

\title{
Estimation of the distance from a surface based on local optic flow divergence
}

\author{
Lucia Bergantin, Thibaut Raharijaona, Franck Ruffier
}

\section{To cite this version:}

Lucia Bergantin, Thibaut Raharijaona, Franck Ruffier. Estimation of the distance from a surface based on local optic flow divergence. 2021 International Conference On Unmanned Aircraft Systems (ICUAS'21), Jun 2021, Athens, Greece. 10.1109/ICUAS51884.2021.9476751 . hal-03293482

\section{HAL Id: hal-03293482 \\ https://hal.science/hal-03293482}

Submitted on 21 Jul 2021

HAL is a multi-disciplinary open access archive for the deposit and dissemination of scientific research documents, whether they are published or not. The documents may come from teaching and research institutions in France or abroad, or from public or private research centers.
L'archive ouverte pluridisciplinaire HAL, est destinée au dépôt et à la diffusion de documents scientifiques de niveau recherche, publiés ou non, émanant des établissements d'enseignement et de recherche français ou étrangers, des laboratoires publics ou privés. 


\section{Estimation of the distance from a surface based on local optic flow divergence}

\author{
Lucia Bergantin \\ Aix-Marseille Université \\ CNRS, ISM \\ 13009 Marseille, France \\ Email: lucia.bergantin@univ-amu.fr
}

\author{
Thibaut Raharijaona \\ Université de Lorraine \\ Arts et Métiers Institute of Technology \\ LCFC, HESAM Université \\ F-57070 Metz, France \\ Email: thibaut.raharijaona@univ-lorraine.fr
}

\author{
Franck Ruffier \\ Aix-Marseille Université \\ CNRS, ISM \\ 13009 Marseille, France \\ Email: franck.ruffier@univ-amu.fr
}

\begin{abstract}
Estimating the distance from a surface is a well-known problem for all kinds of applications involving robots moving in an unknown environment. For flying robots this issue is often coupled with weight constraints, from which the importance of carrying out the estimation of distances with minimalistic equipment. In this study, we present a method to exploit the optic flow divergence cue in order to assess the distance from a surface by means of an Extended Kalman Filter. First, we demonstrated mathematically that the optic flow divergence can be assessed by computing the subtraction between two local optic flow magnitudes. Then, we tested this method on a test bench consisting of two on-the-shelf optic flow sensors performing a back-and-forth oscillatory movement in front of a static or moving panorama. Our findings showed that the optic flow divergence measured as a subtraction of two local optic flow magnitudes was in line with the optic flow divergence computed theoretically under two different lighting conditions. Thus, we were able to use the optic flow divergence measured to assess the distance from the static or moving panorama for low (120lux) and bright (974lux) illuminance respectively. Future work will focus on the implementation of this method on a micro-flier to estimate the distance from a surface, with little mass and computational power.
\end{abstract}

\section{INTRODUCTION}

The problem of distance estimation while navigating in an unknown environment is common to all types of robots. In flying robots, and more specifically in micro-fliers, this need is often coupled with weight constraints. From these considerations stems the importance of carrying out an accurate visual distance estimation by means of minimalistic equipment. Previous authors have suggested the use of stereo vision to avoid obstacles in vehicle environment perception [1, 15] and in flying robots [14], as well as the use of monocular vision for depth perception [20]. All these approaches rely on the use of cameras and often require the use of complex computer vision algorithms. Optic flow (OF) cues have been used on board flying robots to visually control landing with translational OF [18] and with OF divergence [7, 22, 3, 8], to follow uneven terrain [4] or to attempt visual odometry and localisation $[9,10]$ (see [21] for review). Moreover, instabilities have been used to determine the height of flight of a micro-flier by exploiting the linear relation between the oscillation and the fixed control gain [3]. Local OF sensors have been extensively tested in a wide range of lighting conditions for OF based guidance. More generally, the criteria for evaluating the suitability of translational OF sensors for robotic applications include [23]:

- Robustness to light level variations, defined by the number of irradiance decades in which the visual sensor can operate,

- Range of OF angular speeds (or magnitudes) covered, defined by the minimum and maximum values measured,
- Accuracy and precision, defined by systematic errors and coefficients of variation,

- Output refresh rate, defined by the instantaneous output frequency.

To measure the translational $\mathrm{OF}$, a $\mathrm{OF}$ sensor was based on the $\mathrm{M}^{2}$ APix (Michaelis-Menten Auto-adaptive Pixel) retina that can auto-adapt in a 7-decade lighting range [13]. Moreover, it has been demonstrated that a single Local Motion Sensor (LMS) fitted with two auto-adaptive pixels allows to measure an OF range of $\left[50^{\circ} / \mathrm{s}\right.$; $350^{\circ} / \mathrm{s}$ ] despite variations in lighting conditions from $\sim 50 \mathrm{lux}$ to $10,000 l u x$ [5]. A similar OF range of $\left[50^{\circ} / s ; 250^{\circ} / s\right]$ (i.e., $0.7-$ decade) was measured with a semi-panoramic artificial eye, called CurvACE [6]. The OF range can also be measured for outdoor flights, as in [19]. In [17], LMSs were used in front of a moving panorama to measure translational OF.

Small self-oscillatory movements have been observed in honeybees flying in horizontal [11] and vertical tunnels [16]. These self-induced oscillations are different from side-to-side parallax movements observed in insects (more specifically in locusts [2] and in praying mantis [12]). These oscillations generate a pattern of expansions and contractions in the OF vector field, known as OF divergence. The changes in the vertical speed and in the height of flight due to the oscillations make both variables locally observable [8]. Therefore, by having a device performing backand-forth oscillatory movements in front of a surface, the local observability of its speed and distance from the surface is ensured. Taking inspiration from honeybees, in this study we exploit the $\mathrm{OF}$ divergence cue to visually estimate the distance from a static or moving surface. In previous studies, authors have used cameras to assess the OF divergence and in some cases to estimate the distance from a surface using an Extended Kalman Filter (EKF) on board a flying robot [8]. In this study, we assessed the distance from a static or moving surface by measuring the OF divergence solely based on the subtraction of the magnitudes of two local OF sensors.

In section II, we demonstrate mathematically that the OF divergence can be measured solely based on the subtraction of the magnitudes of two local OF sensors: this subtraction results in the OF divergence even in front of a static or a moving surface. In section III, we present the test bench consisting of two OF sensors placed on a slider performing a back-and-forth oscillatory movement in front of a panorama. In section IV, we show experimentally that the signal obtained was equivalent to the OF divergence computed theoretically. Then, the signal was conveyed to the EKF to assess the distance from the static or moving panorama under two different lighting conditions. 

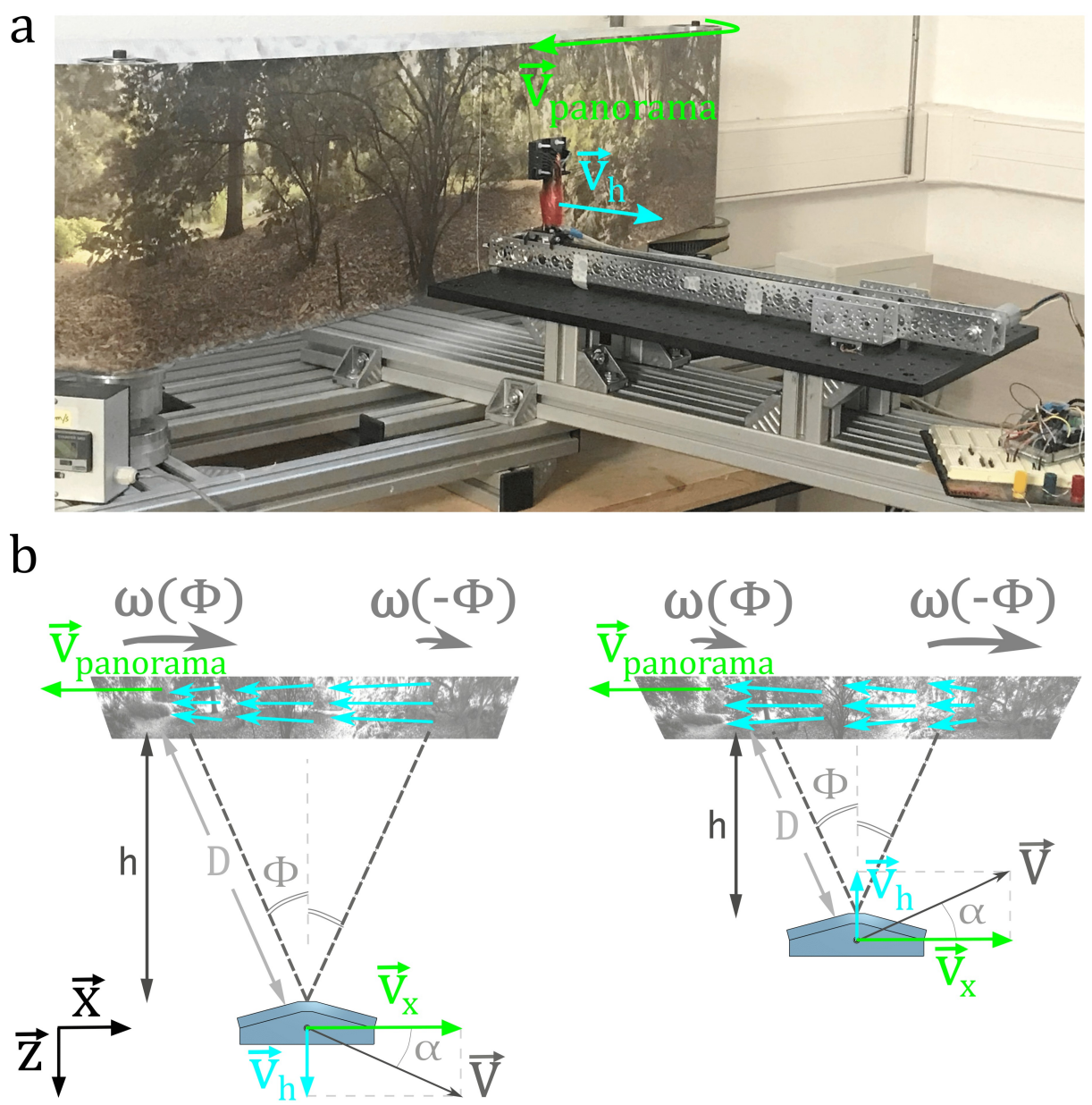

Fig. 1: (a) The test bench consisted of two OF sensors set on a chariot at $\phi$ and $-\phi$ (with $\phi=15^{\circ}$ ) with respect to the horizontal axis $z$ and placed on a slider in front of a panorama. The DC motor connected at the end of the slider opposite to the panorama induced a back-and-forth oscillatory movement on the chariot on the horizontal axis $z$. The direction of the chariot's movement was associated with the velocity $v_{h}$, represented in blue. The panorama moved on the $x$ axis at a varying velocity $V_{\text {panorama }}$ (in green). A lidar placed on the chariot measured the distance $D \cdot \cos (\phi)$ to the panorama and hence provided the ground truth for the experiment. (b) The two OF sensors measured the OF magnitudes $\omega(\phi)$ and $\omega(-\phi)$, respectively. When the DC motor induced a velocity $v_{h}$ in the direction of the $z$ axis, the movement of the two OF sensors coupled with the movement of the panorama mimicked an ascending flight above a static surface. The resulting velocity $V$ was oriented upward with an angle $\alpha$ and a contraction was perceived on the OF vector field. When the DC motor induced a velocity $v_{h}$ in the opposite direction with respect to the $z$ axis, the movement of the two OF sensors coupled with the movement of the panorama mimicked a descending flight above a static surface. In this case, $V$ was oriented downward with an angle $\alpha$ and an expansion was perceived on the OF vector field.

Due to their low weight, OF sensors are particularly interesting for flying robotic applications. Thus, we plan to test the method presented to estimate the distance of a flying robot at about one meter from a surface.

\section{THE OPTIC FLOW DIVERGENCE}

\section{A. Definition of the local optic flow divergence}

Oscillatory movements generate a sequence of expansions and contractions in the $\mathrm{OF}$ vector field. This pattern is known as $\mathrm{OF}$ divergence and is superimposed on the translational OF component of the OF vector field. The OF divergence is defined as the ratio of the velocity in the direction normal to a surface $v_{h}$ and the distance from the surface $h$ :

$$
\omega_{D I V}^{t h}=\frac{v_{h}}{h}
$$

These oscillations make the state vector of the oscillating system locally observable [8] and hence allow to assess the distance from the surface by means of an estimator, i.e. an EKF. The OF divergence can be used by flying robots as an additional visual cue for distance estimation purposes.

B. True local optic flow divergence measured by only two optic flow sensors

We mathematically demonstrate here that the local OF divergence $\omega_{\text {div }}^{\text {meas }}$ can be computed as the subtraction between the magnitudes of two OF sensors' outputs.

Proof. To compute the OF divergence, we consider the case of a device equipped with two OF sensors oriented forward at $\phi$ and $-\phi$ with respect to the horizontal axis $z$ and placed in front of 
a surface. The device moves forward oscillating back-and-forth in front of the surface, that moves at a speed $-v_{x}$. The OF divergence can be measured as follows

$$
\omega_{D I V}^{\text {meas }}=\omega(\phi)-\omega(-\phi)
$$

with $\omega(\phi)$ and $\omega(-\phi)$ the OF magnitudes measured by the two OF sensors respectively. By definition, the OF due to the translation movement of the surface can be written as follows

$$
\omega(\phi)=\frac{\|\vec{V}\|}{D} \sin (\widehat{\vec{D}, \vec{V}})
$$

with

$$
(\widehat{\vec{D}, \vec{V}})=\frac{\pi}{2}-\phi+\alpha
$$

We can express the two components of the velocity vector $\vec{V}$ of the device in front of the surface as:

$$
\begin{aligned}
& v_{x}=\|\vec{V}\| \cdot \cos \alpha \\
& v_{h}=\|\vec{V}\| \cdot \sin \alpha
\end{aligned}
$$

with

$$
\|\vec{V}\|=\sqrt{v_{x}^{2}+v_{h}^{2}}
$$

from which we obtain:

$$
\begin{aligned}
\cos \alpha & =\frac{v_{x}}{\sqrt{v_{x}^{2}+v_{h}^{2}}} \\
\sin \alpha & =\frac{v_{h}}{\sqrt{v_{x}^{2}+v_{h}^{2}}}
\end{aligned}
$$

Thus

$$
\begin{aligned}
\omega(\phi) & =\frac{\|\vec{V}\|}{D} \sin (\widehat{\vec{D}, \vec{V}}) \\
& =\frac{\sqrt{v_{x}^{2}+v_{h}^{2}}}{D} \cdot \sin \left(\frac{\pi}{2}-\phi+\alpha\right) \\
& =\frac{\sqrt{v_{x}^{2}+v_{h}^{2}}}{D} \cdot\left(\sin \left(\frac{\pi}{2}-\phi\right) \cos \alpha+\cos \left(\frac{\pi}{2}-\phi\right) \sin \alpha\right) \\
& =\frac{\sqrt{v_{x}^{2}+v_{h}^{2}}}{D} \cdot\left(\sin \left(\frac{\pi}{2}-\phi\right) \frac{v_{x}}{\sqrt{v_{x}^{2}+v_{h}^{2}}}\right. \\
& \left.+\cos \left(\frac{\pi}{2}-\phi\right) \frac{v_{h}}{\sqrt{v_{x}^{2}+v_{h}^{2}}}\right) \\
& =\frac{v_{x}}{D} \sin \left(\frac{\pi}{2}-\phi\right)+\frac{v_{h}}{D} \cos \left(\frac{\pi}{2}-\phi\right) \\
& =\frac{v_{x}}{D} \sin \left(\frac{\pi}{2}-\phi\right)+\frac{v_{h}}{D} \sin \phi \\
& =\frac{\left\|\overrightarrow{v_{x}}\right\|}{D} \sin \left(\widehat{\vec{D}, \overrightarrow{v_{x}}}\right)+\frac{\left\|\overrightarrow{v_{h}}\right\|}{D} \sin \left(\widehat{\vec{D}, \overrightarrow{v_{h}}}\right)
\end{aligned}
$$

We can then express $\omega(-\phi)$ and $\omega(\phi)$ as:

$$
\begin{gathered}
\omega(-\phi)=\frac{v_{x}}{D} \sin \left(\frac{\pi}{2}-\phi\right)-\frac{v_{h}}{D} \sin \phi \\
\omega(\phi)=\frac{v_{x}}{D} \sin \left(\frac{\pi}{2}-\phi\right)+\frac{v_{h}}{D} \sin \phi
\end{gathered}
$$

Thus, the OF divergence can be computed as

$$
\omega(\phi)-\omega(-\phi)=2 \cdot \frac{v_{h}}{D} \cdot \sin (\phi)
$$

where $h=D \cdot \cos (\phi)$ is the distance from the surface.

$$
\omega(\phi)-\omega(-\phi)=2 \cdot \frac{v_{h}}{h} \cdot \sin (\phi) \cdot \cos (\phi)
$$

Since $\sin (\phi) \cdot \cos (\phi)=\frac{1}{2} \cdot \sin (2 \cdot \phi)$, we can express equation (14) as follows:

$$
\omega_{D I V}^{\text {meas }}=\omega(\phi)-\omega(-\phi)=\frac{v_{h}}{h} \cdot \sin (2 . \phi)
$$

where $\omega$ is the OF magnitude, $\phi$ is the visual direction of the OF sensor with respect to the axis $z$ and $h$ is the distance from the panorama. The maximum sensitivity of such a local OF divergence device corresponds to a $\mathrm{OF}$ sensor orientation of $\phi=45^{\circ}$.

\section{Materials And Methods}

To show that it is possible for an oscillating system to retrieve its distance from a surface solely on the basis of OF cues, we built a test bench consisting of two OF sensors set at fixed angles $\phi$ and $-\phi$ which performed a back-and-forth oscillatory movement in front of a panorama. Since the system was locally observable due to the oscillations, it was possible to estimate the distance from the static or moving panorama by means of the EKF. The EKF received as measurement the OF divergence computed as in equation (2), with $\omega(\phi)$ and $\omega(-\phi)$ the OF magnitudes measured by the two OF sensors. To test the robustness of this method, we considered two lighting conditions: bright $\left(974 \mathrm{lux}, 6.95 \cdot 10^{-5} \mathrm{~W} / \mathrm{cm}^{2}\right)$ and low (120lux, $5.42 \cdot 10^{-6} \mathrm{~W} / \mathrm{cm}^{2}$ ) illuminance, respectively. In the test bench, $V_{x}=-V_{\text {panorama }}$ because the movement of the panorama mimicked the flight forward above a surface of the system to which the OF sensors were attached.

\section{A. The test bench}

The test bench built consisted of two OF sensors set on a chariot at $\phi$ and $-\phi$ (with $\phi=15^{\circ}$ ) with respect to the horizontal axis $z$ and placed on a slider in front of a panorama, as shown in Figure 1. A DC motor was connected at the end of the slider opposite to the panorama and induced a back-and-forth movement on the chariot along the slider on the horizontal axis $z$. The panorama moved on the $x$ axis at a varying velocity $V_{\text {panorama }}$. A lidar set on the chariot provided the ground truth measuring the distance $D \cdot \cos (\phi)$ from the panorama. The back-and-forth movement of the OF sensors reproduced the self-oscillations observed in honeybees, while the movement of the panorama mimicked the motion forward above a surface. Synchronization was guaranteed by the use of an interface that initialized the OF sensors, the lidar and the DC motor at the same time. More specifically, we used two Flow Deck V2 from https://www.bitcraze.io/. On each deck were set a PixArt PMW3901 OF sensor and a VL53L1x ToF lidar. The sensors were connected to an Arduino DUE board. To compute the ground truth, we relied on the output of the lidar on the left. The interface MyViz was used for synchronization purposes and to change the frequency of the chariot's oscillation when required.

\section{B. Calibration of the optic flow sensors}

To compute the OF divergence, we relied on the OF magnitudes $\omega(\phi)$ and $\omega(-\phi)$ measured by the two OF sensors set on the chariot. The OF sensors' raw outputs were given in [pixel/s] and thus needed to be calibrated to be expressed in $[\mathrm{rad} / \mathrm{s}]$. To find the calibration coefficients, a position on the slider was chosen $(0.14 \mathrm{~m})$. One OF sensor was kept stationary with $\phi=0^{\circ}$ with respect to the axis $z$, while the panorama moved with nine values of velocity 
$V_{\text {panorama }}($ from $0.2 \mathrm{~m} / \mathrm{s}$ up to $1 \mathrm{~m} / \mathrm{s}$ with a step of $0.1 \mathrm{~m} / \mathrm{s}$ ). For each $V_{\text {panorama }}$, were computed the theoretical translational $\mathrm{OF}$ $w_{T}^{t h}$ and the average of the measured translational OF $\bar{w}_{T}^{\text {meas }}$. Nine points of coordinates $\left(w_{T}^{\text {th }}, \bar{w}_{T}^{\text {meas }}\right)$ were obtained and used to fit an affine line $\bar{w}_{T}^{\text {meas }}=m \cdot w_{T}^{t h}+q$, where $m$ and $q$ were the calibration coefficients. This procedure was applied to the two OF sensors separately.

\section{The model of the test bench}

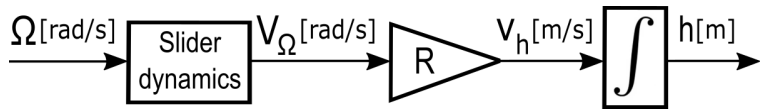

Fig. 2: Block diagram of the test bench system. The system of the test bench received as input the velocity setpoint of the DC motor $\Omega[\mathrm{rad} / \mathrm{s}]$ and as output the distance $h[\mathrm{~m}]$ of the two OF sensors from the panorama. More specifically, the slider dynamics received $\Omega$ as input and gave the velocity induced on the chariot by the DC motor $V_{\Omega}[\mathrm{rad} / \mathrm{s}]$ as output. $V_{\Omega}$ was scaled by the radius $R$ of the pulley connecting the DC motor to the chariot in order to compute $v_{h}[\mathrm{~m} / \mathrm{s}] . v_{h}$ was then integrated in order to obtain $h$.

The system of the test bench can be represented as the block diagram in Figure 2. The system's input was the velocity setpoint of the DC motor $\Omega$, while its output was the distance $h$ of the two OF sensors from the panorama. The slider dynamics represents the motion control system, as to say a feedback loop over a PID and the motor dynamics. The slider dynamics received as input the DC motor's velocity setpoint $\Omega$ in $[\mathrm{rad} / \mathrm{s}]$ and gave as output the velocity induced by the DC motor on the chariot $V_{\Omega}$ in $[\mathrm{rad} / \mathrm{s}]$. $V_{\Omega}$ was scaled by the radius $R$ (with $R=0.012 m$ ) of the pulley connecting the DC motor to the chariot in order to compute $v_{h}$, which was then integrated to compute $h$. We identified the slider's system between the setpoint input $\Omega$ and the chariot's displacement $h$ in [m] using a transfer function that can be expressed as

$$
G(s)_{\text {Slider }}=\frac{Z(s)}{U(s)}=\frac{0.3498}{s(s+54.27)}
$$

The system's state space representation can then be expressed as

$$
\left\{\begin{array}{c}
\dot{X}=A \cdot X+B \cdot u=\left[\begin{array}{cc}
0 & 1 \\
0 & -54.27
\end{array}\right] X+\left[\begin{array}{c}
0 \\
0.3498
\end{array}\right] u \\
Y=C \cdot X+D \cdot u=\left[\begin{array}{ll}
1 & 0 \\
0 & 1
\end{array}\right] X
\end{array}\right.
$$

where $\mathrm{u}$ is the velocity setpoint $\Omega$ and $X=\left[h ; v_{h}\right]$ is the state vector. It is important to notice that the measurement equation of the model is nonlinear, since it can be expressed as

$$
Y=\omega_{D I V}=\frac{v_{h}}{h}
$$

This is the reason why the use of an EKF is necessary. Once defined the model of the test bench system in equation (18), we proceeded to its discretization (see Appendix A for the EKF calculations). The EKF received as input the velocity setpoint of the DC motor $\Omega$ and as measurement the OF divergence measured.

\section{RESUlts}

To show experimentally that the measured OF divergence obtained as in equation (2) was equivalent to the theoretical $\mathrm{OF}$ divergence computed as in equation (16), several datasets were collected under different conditions. The two OF sensors performed a back-and-forth oscillatory movement in front of the panorama, which was moving with different values of velocity $V_{\text {panorama }}$ $(0 \mathrm{~m} / \mathrm{s}, 0.25 \mathrm{~m} / \mathrm{s}, 0.5 \mathrm{~m} / \mathrm{s}$ and $0.75 \mathrm{~m} / \mathrm{s})$. The panorama speed $V_{\text {panorama }}$ can not exceed $0.75 \mathrm{~m} / \mathrm{s}$ steadily because of mechanical constraints. For each $V_{\text {panorama }}$, four oscillation frequencies were considered: $0.25 \mathrm{~Hz}, 0.5 \mathrm{~Hz}, 0.75 \mathrm{~Hz}$ and $1 \mathrm{~Hz}$. The maximum peak-to-peak amplitude was reached for $0.25 \mathrm{~Hz}$ (see details in caption of the Figure 3). For oscillation frequencies below $1 \mathrm{~Hz}$, the oscillation amplitude was too small to allow an effective estimation of the distance from the panorama with the current setup. To test the robustness of the method, the datasets were taken under two different lighting conditions: bright $\left(974 l u x, 6.95 \cdot 10^{-5} \mathrm{~W} / \mathrm{cm}^{2}\right)$ and low (120lux, $\left.5.42 \cdot 10^{-6} \mathrm{~W} / \mathrm{cm}^{2}\right)$ illuminance, respectively. For each dataset we subtracted the OF magnitudes measured by the two OF sensors as in equation (2), obtaining the corresponding OF divergence $\omega_{\text {div }}^{\text {meas }}$. In parallel, we computed the theoretical OF divergence $\omega_{d i v}^{t h}$ as in equation (16). To compare $\omega_{d i v}^{\text {meas }}$ and $\omega_{d i v}^{t h}$, all the values of $\omega_{\text {div }}^{\text {meas }}$ measured for all the oscillation frequencies for the panorama moving with a given $V_{\text {panorama }}$ under a set lighting condition were put together and plotted in comparison with the corresponding $\omega_{d i v}^{t h}$. Figure 3 shows experimentally that the subtraction between the OF magnitudes obtained by means of the two OF sensors can measure the OF divergence as demonstrated mathematically in section II-B. The plots show that $\omega_{\text {div }}^{\text {meas }}$ and $\omega_{\text {div }}^{\text {th }}$ were in the same range of values for every set of conditions. The Median Absolute Deviation (MAD) was low for every case considered, ranging between $0.25 \mathrm{rad} / \mathrm{s}$ and $0.38 \mathrm{rad} / \mathrm{s}$ (see Table I). Thus, the OF divergence measured $\omega_{d i v}^{\text {meas }}$ can be considered in line with $\omega_{d i v}^{t h}$ under every set of conditions analysed and hence can be given as measurement to an EKF to estimate the distance from a static or moving surface.

\begin{tabular}{|l|c|c|}
\hline Average MAD of the OF divergence & low light & bright light \\
\hline$V_{\text {panorama }}=0 \mathrm{~m} / \mathrm{s}$ & $0.27 \mathrm{rad} / \mathrm{s}$ & $0.27 \mathrm{rad} / \mathrm{s}$ \\
$V_{\text {panorama }}=0.25 \mathrm{~m} / \mathrm{s}$ & $0.26 \mathrm{rad} / \mathrm{s}$ & $0.25 \mathrm{rad} / \mathrm{s}$ \\
$V_{\text {panorama }}=0.5 \mathrm{~m} / \mathrm{s}$ & $0.29 \mathrm{rad} / \mathrm{s}$ & $0.30 \mathrm{rad} / \mathrm{s}$ \\
$V_{\text {panorama }}=0.75 \mathrm{~m} / \mathrm{s}$ & $0.38 \mathrm{rad} / \mathrm{s}$ & $0.34 \mathrm{rad} / \mathrm{s}$ \\
\hline
\end{tabular}

TABLE I: Table of the average MAD obtained for the comparison of $\omega_{\text {div }}^{\text {meas }}$ and $\omega_{d i v}^{t h}$ for four values of $V_{\text {panorama }}(0 \mathrm{~m} / \mathrm{s}, 0.25 \mathrm{~m} / \mathrm{s}$, $0.5 \mathrm{~m} / \mathrm{s}$ and $0.75 \mathrm{~m} / \mathrm{s})$ under bright $\left(974 l u x, 6.95 \cdot 10^{-5} \mathrm{~W} / \mathrm{cm}^{2}\right)$ and low $\left(120 \mathrm{lux}, 5.42 \cdot 10^{-6} \mathrm{~W} / \mathrm{cm}^{2}\right)$ illuminance, respectively. The values of the average MAD ranged between $0.25 \mathrm{rad} / \mathrm{s}$ and $0.38 \mathrm{rad} / \mathrm{s}$.

Since we showed that the OF divergence can be measured reliably as the subtraction of two OF magnitudes, we used it to estimate the distance from the static or moving panorama by means of the EKF. The OF sensors moved back and forth in front of the panorama for $10 \mathrm{~s}$ with a frequency of $0.5 \mathrm{~Hz}$. This was done for four values of $V_{\text {panorama }}(0 \mathrm{~m} / \mathrm{s}, 0.25 \mathrm{~m} / \mathrm{s}, 0.5 \mathrm{~m} / \mathrm{s}$ and $0.75 \mathrm{~m} / \mathrm{s})$ under bright and low illuminance. Figure 4.a shows the results obtained under bright illuminance. The estimates of the distance from the panorama $\hat{h}$ converged quickly (about $2 s$ ) to the ground truth $h$ for $V_{\text {panorama }}$ equal to $0 \mathrm{~m} / \mathrm{s}, 0.25 \mathrm{~m} / \mathrm{s}$ and $0.5 \mathrm{~m} / \mathrm{s}$. For $V_{\text {panorama }}$ equal to $0.75 \mathrm{~m} / \mathrm{s}$ the converging time increased slightly (about $3 s$ ). In every case, the OF divergence was a sinusoidal signal due to the pattern of expansion and contraction in the OF vector field induced by the oscillatory movement. The OF divergence measured presented noise, due to the $\mathrm{OF}$ measurement noise, the limitations in the view-field of PixArt OF sensors and the mechanical noise caused by the movement of the chariot on the slider. In general, 

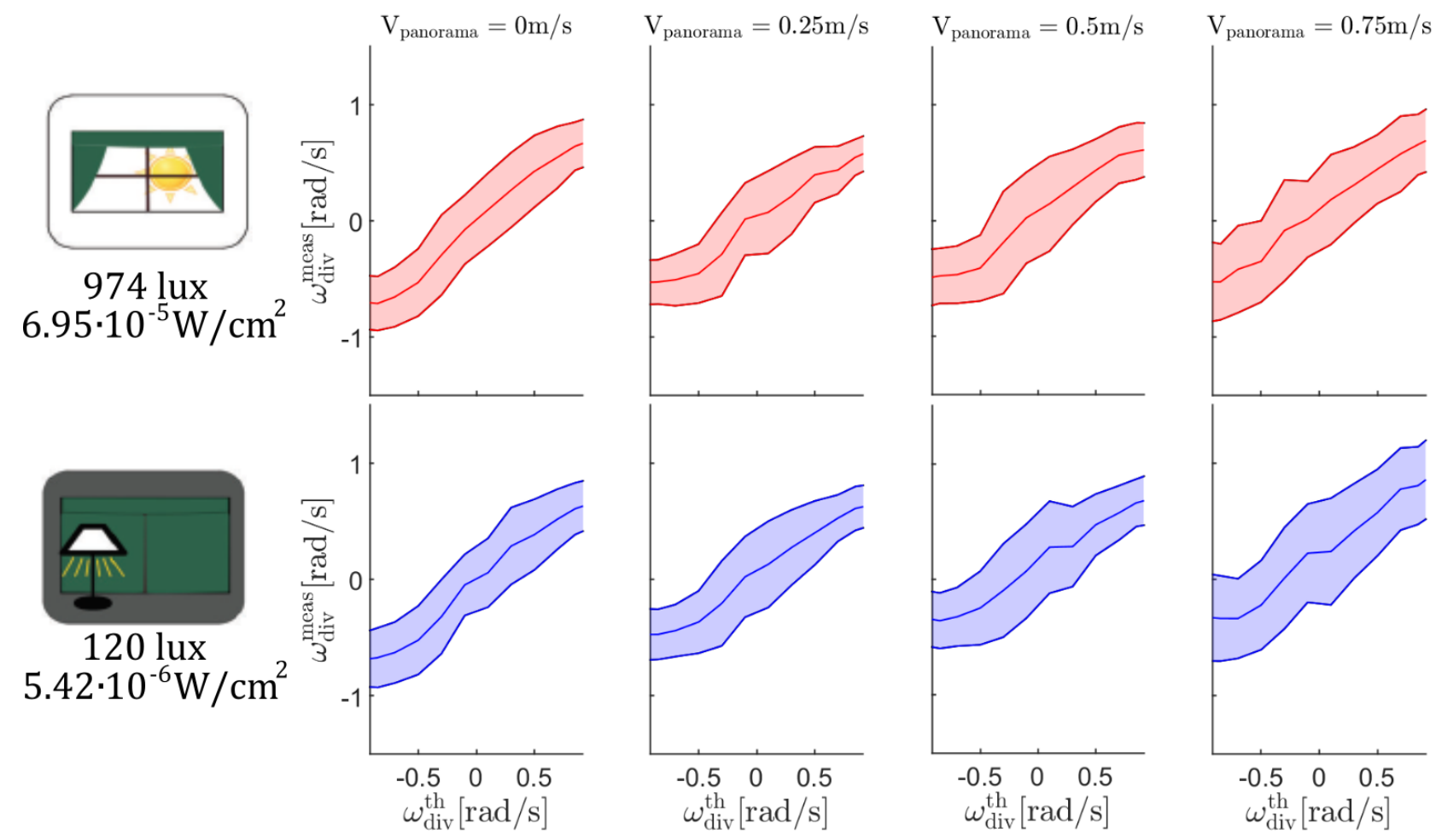

Fig. 3: The plots show a linear relation between $\omega_{d i v}^{\text {meas }}$ and $\omega_{d i v}^{\text {th }}$ and are therefore an experimental counterpart of the mathematical proof shown in section II-B. The Median Absolute Deviation (MAD) of each plot was low (see Table I). The OF divergence $\omega_{\text {div }}^{\text {meas }}$ was measured in front of the static or moving panorama under two lighting conditions: bright $\left(974 \mathrm{lux}, 6.95 \cdot 10^{-5} \mathrm{~W} / \mathrm{cm}^{2}\right)$ and low $(120 \mathrm{lux}$, $\left.5.42 \cdot 10^{-6} \mathrm{~W} / \mathrm{cm}^{2}\right)$ illuminance, respectively. For each value of $V_{\text {panorama }}(0 \mathrm{~m} / \mathrm{s}, 0.25 \mathrm{~m} / \mathrm{s}, 0.5 \mathrm{~m} / \mathrm{s}$ and $0.75 \mathrm{~m} / \mathrm{s})$ four values of oscillation frequency were considered $(0.25 \mathrm{~Hz}, 0.5 \mathrm{~Hz}, 0.75 \mathrm{~Hz}$ and $1 \mathrm{~Hz})$. For all datasets, the starting position of the chariot on the slider was $0.14 \mathrm{~m}$. The peak-to-peak amplitude covered by the chariot on the slider ranged from the starting position to about $0.3 \mathrm{~m}$ for a frequency of $0.25 \mathrm{~Hz}, 0.25 \mathrm{~m}$ for $0.5 \mathrm{~Hz}, 0.2 \mathrm{~m}$ for $0.75 \mathrm{~Hz}$ and $0.17 \mathrm{~m}$ for $1 \mathrm{~Hz}$. The theoretical OF divergence $\omega_{\text {div }}^{\text {th }}$ was computed for every dataset. The values of $\omega_{\text {div }}^{\text {meas }}$ measured for all the oscillation frequencies for a velocity $V_{\text {panorama }}$ under a given lighting condition were put together and plotted in comparison with the corresponding $\omega_{d i v}^{t h}$.In each plot, the median values of $\omega_{d i v}^{t h}$ and the curves representing its MAD are shown to display the range of values measured. The subtraction between the OF magnitudes obtained by means of the two OF sensors can measure reliably a stimuli of OF divergence between $-0.93 \mathrm{rad} / \mathrm{s}$ and $1.3 \mathrm{rad} / \mathrm{s}$.

the noise increased with $V_{\text {panorama }}$. The presence of a higher noise magnitude explained the slightly higher convergence time for $V_{\text {panorama }}=0.75 \mathrm{~m} / \mathrm{s}$. Figure $4 . \mathrm{b}$ shows the results obtained under low illuminance. As in the case of bright illuminance, the estimates of the distance from the panorama $\hat{h}$ converged quickly (about $2 s$ ) to the ground truth $h$ for $V_{\text {panorama }}$ equal to $0 \mathrm{~m} / \mathrm{s}$ and $0.25 \mathrm{~m} / \mathrm{s}$. For $V_{\text {panorama }}$ equal to $0.5 \mathrm{~m} / \mathrm{s}$ and $0.75 \mathrm{~m} / \mathrm{s}$ the converging time increased (about $3 s$ ) due to higher noise magnitudes. The noise was present in every case and increased with $V_{\text {panorama }}$.

To compare the estimation performances of the method under the two lighting conditions, an analysis of the average error of the estimates of the distance from the panorama $\hat{h}$ after the convergence to the ground truth $h$ was performed. The results are shown in Table II.

We considered as time of convergence $3 s$ in order to include all the datasets analysed. The average errors computed ranged between $0.31 \%$ and $15.73 \%$. The errors computed under low illuminance were slightly higher than those obtained under bright illuminance. The only exception was $V_{\text {panorama }}=0.75 \mathrm{~m} / \mathrm{s}$, for which the average error under bright illuminance was about $3 \%$ higher than under low illuminance. The results obtained under bright and low

\begin{tabular}{|l|c|c|}
\hline Estimation average errors & low light & bright light \\
\hline$V_{\text {panorama }}=0 \mathrm{~m} / \mathrm{s}$ & $4.49 \%$ & $0.31 \%$ \\
$V_{\text {panorama }}=0.25 \mathrm{~m} / \mathrm{s}$ & $15.73 \%$ & $12.09 \%$ \\
$V_{\text {panorama }}=0.5 \mathrm{~m} / \mathrm{s}$ & $12.03 \%$ & $3.29 \%$ \\
$V_{\text {panorama }}=0.75 \mathrm{~m} / \mathrm{s}$ & $5.41 \%$ & $8.29 \%$ \\
\hline
\end{tabular}

TABLE II: Table of the average errors of the estimates of the distance from the panorama $\hat{h}$ after the convergence to the ground truth $h$ for four values of $V_{\text {panorama }}(0 \mathrm{~m} / \mathrm{s}, 0.25 \mathrm{~m} / \mathrm{s}, 0.5 \mathrm{~m} / \mathrm{s}$ and $0.75 \mathrm{~m} / \mathrm{s}$ ) under bright and low illuminance (974lux and 120lux, respectively). We computed the average error between $3 s$ and $10 \mathrm{~s}$ to guarantee that all the datasets converged. The average errors ranged between $0.31 \%$ and $15.73 \%$.

illuminance can be considered similar. Thus, our findings show that $\omega_{\text {div }}^{\text {meas }}$ can be used to estimate reliably the distance from a surface under both lighting conditions.

\section{CONCLUSION}

The observation of self-induced oscillatory movements in honeybees $[11,16]$ has led to consider the use of an additional OF cue: the OF divergence. The presence of oscillations makes the oscillating 
a

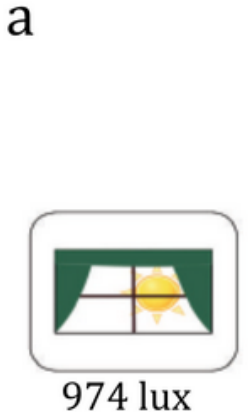

$6.95 \cdot 10^{-5} \mathrm{~W} / \mathrm{cm}^{2}$
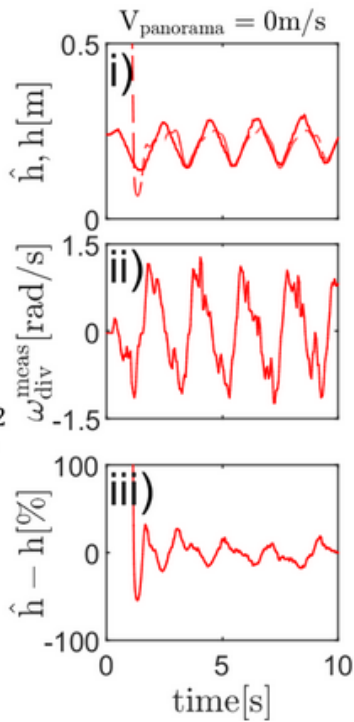

$\mathrm{b}$



$120 \operatorname{lux}$
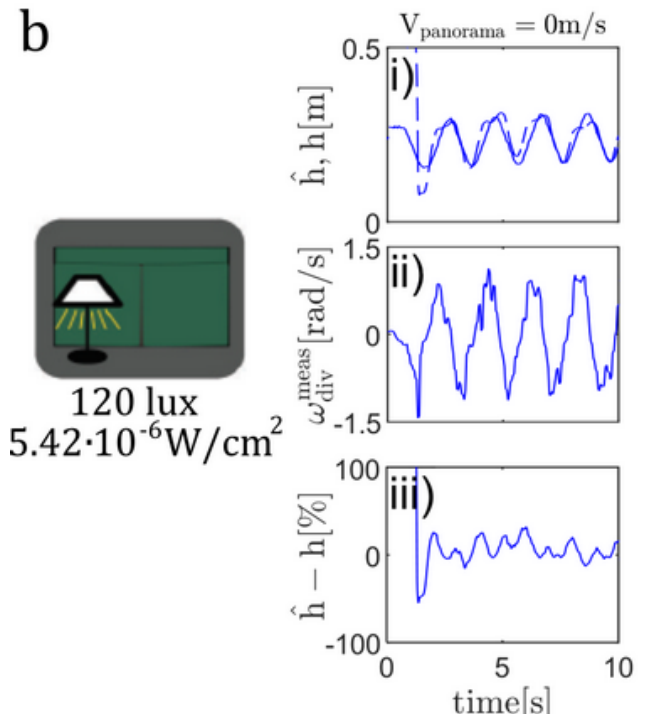
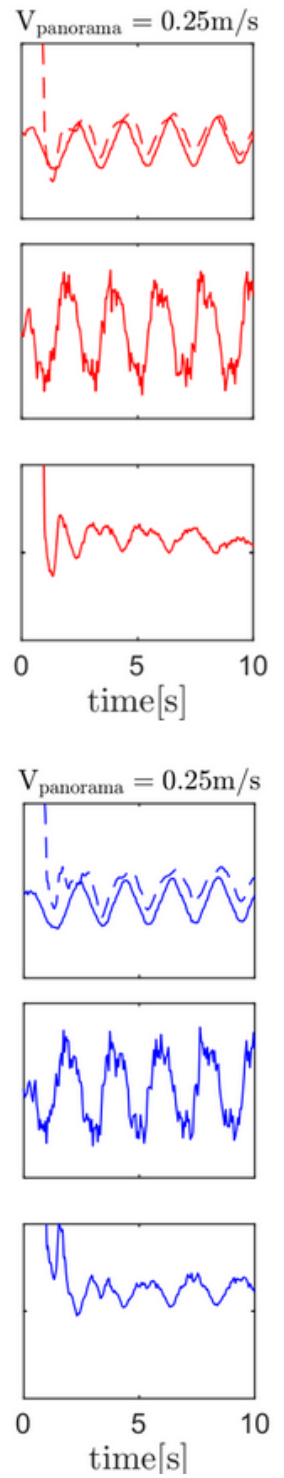
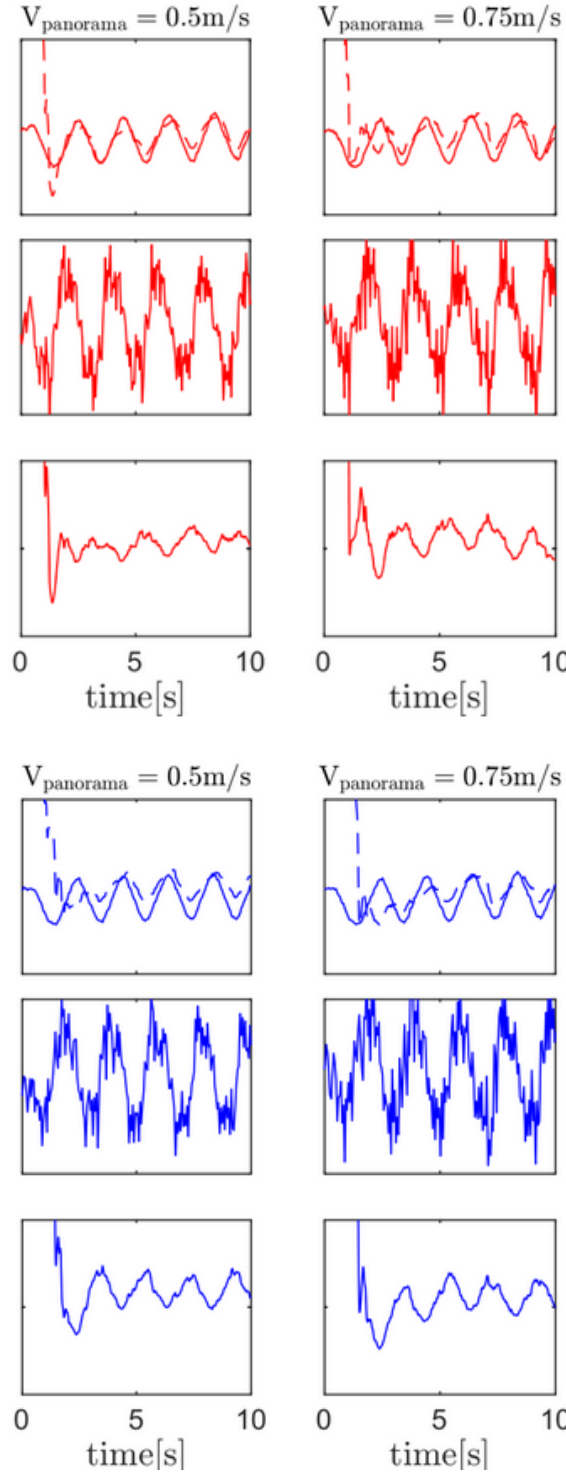

Fig. 4: The OF divergence was measured with an oscillation frequency of $0.5 \mathrm{~Hz}$ under (a) bright $\left(974 l u x, 6.95 \cdot 10^{-5} \mathrm{~W} / \mathrm{cm}^{2}\right)$ and $(\mathrm{b})$ low $\left(120 \mathrm{lux}, 5.42 \cdot 10^{-6} \mathrm{~W} / \mathrm{cm}^{2}\right)$ illuminance in front of the panorama moving with four values of $V_{\text {panorama }}(0 \mathrm{~m} / \mathrm{s}, 0.25 \mathrm{~m} / \mathrm{s}, 0.5 \mathrm{~m} / \mathrm{s}$ and $0.75 \mathrm{~m} / \mathrm{s}$ ). i) The estimates of the distance from the panorama $\hat{h}$ converged quickly (from about $2 s$ to $3 s$ ) to the ground truth $h$ for every value of $V_{\text {panorama }}$. The distance $h$ covered by the chariot on the slider ranged between $0.14 \mathrm{~m}$ and $0.25 \mathrm{~m}$. ii) In every case, the OF divergence measured $\omega_{\text {div }}^{\text {meas }}$ was a sinusoidal signal. The noise magnitude increased with $V_{\text {panorama }}$ and in general was slightly higher for datasets taken under low illuminance. The higher noise magnitude resulted in a slightly higher convergence time in the case of $V_{\text {panorama }}$ equal to $0.75 \mathrm{~m} / \mathrm{s}$ under bright illuminance and $0.5 \mathrm{~m} / \mathrm{s}$ and $0.75 \mathrm{~m} / \mathrm{s}$ under low illuminance. iii) In (a.iii), the average error values computed after convergence were $0.31 \%, 12.09 \%, 3.29 \%$ and $8.29 \%$, respectively. In (b.iii), the average error values computed after convergence were $4.49 \%, 15.73 \%, 12.03 \%$ and $5.41 \%$, respectively (see Table (II)).

system always locally observable and hence opens the possibility of using the OF divergence cue to assess the distance from a surface, regardless of the maneuver performed. OF divergence has already been used to visually control landing in a micro-flier [8].

In this study, we exploit the OF divergence cue in order to assess the distance from a surface without the need of previous knowledge of the environment or of emissive sensors. The OF divergence can be computed as the subtraction between the magnitudes measured by two OF sensors. To test this method a test bench was built, con- sisting of two OF sensors performing a back-and-forth oscillatory movement in front of a static or moving panorama. The OF sensors were set on a chariot at an angle of $15^{\circ}$ and $-15^{\circ}$ respectively from the normal to the panorama. The chariot was placed on a slider and was actuated by a DC motor in order to move back and forth along the slider in front of the panorama. To test the robustness of this method, datasets were taken while the panorama was moving at different velocities under bright and low lighting conditions.

Our findings show that it is possible to compute reliably the OF 
divergence between $-0.93 \mathrm{rad} / \mathrm{s}$ and $1.3 \mathrm{rad} / \mathrm{s}$ as the subtraction of OF magnitudes measured by two OF sensors. Hence, the OF divergence measured this way can be used to assess the distance from the panorama by means of an EKF. We successfully tested this method for distances ranging between $0.14 \mathrm{~m}$ and $0.25 \mathrm{~m}$. The estimates of the distance from the panorama were generally more accurate for lower velocities of the panorama, due to a lower noise magnitude. In every case considered, the estimates of the distance from the panorama converged within $3 s$ to the ground truth. The ground truth was obtained by a separate mean: a lidar placed on the chariot. The lighting conditions tested did not influence the results obtained.

Given the low weight of the OF sensors and the low computational power required to compute the OF divergence, this method can be easily implemented on flying robots and more specifically on microfliers. In this study, we tested the method presented only for a small range of distances, all of them fairly short if considering the flight of a micro-flier. Our results were promising and showed that it is worthwhile to test the method further. Future work will include an analysis of the estimation of the distance from the panorama for a wider range of oscillation frequencies and of $V_{\text {panorama }}$ to prepare for the implementation of this method on a micro-flier. Moreover, we plan to use OF sensors with wider optical aperture lenses to expand the range of lighting conditions. Ultimately, we plan to test the presented method to estimate the distance of a flying robot at about one meter from a surface.

\section{ACKNOWLEDGMENT}

We thank J. Diperi and J.M. Ingargiola for their help in the design of the test bench. Financial support was provided via a ProxyLearn project grant to F.R. from the ANR (Astrid Program). The participation of L.B. in this research project was supported by a joint $\mathrm{PhD}$ grant from the Délégation Générale de l'Armement (DGA) and Aix Marseille University. L.B, T.R. and F.R. were also supported by Aix Marseille University and the CNRS (Life Science, Information Science and Engineering Science \& Technology Institutes).

\section{REFERENCES}

[1] Massimo Bertozzi et al. "Stereo vision-based vehicle detection". In: Proceedings of the IEEE Intelligent Vehicles Symposium 2000 (Cat. No. O0TH8511). IEEE. 2000, pp. 39-44.

[2] TS Collett. "Peering-a locust behaviour pattern for obtaining motion parallax information". In: Journal of experimental Biology 76.1 (1978), pp. 237-241.

[3] Guido CHE de Croon. "Monocular distance estimation with optical flow maneuvers and efference copies: a stability-based strategy". In: Bioinspiration \& biomimetics 11.1 (2016), p. 016004.

[4] Fabien Expert and Franck Ruffier. "Flying over uneven moving terrain based on optic-flow cues without any need for reference frames or accelerometers". In: Bioinspiration and Biomimetics 10 (2015). DOI: 10.1088/1748-3190/10/2/ 026003.

[5] Fabien Expert, Stéphane Viollet, and Franck Ruffier. "A mouse sensor and a 2-pixel motion sensor exposed to continuous illuminance changes". In: IEEE Sensors (2011), pp. 974977. ISSN: 1930-0395. DOI: 10.1109/ICSENS.2011.6127002.

[6] Dario Floreano et al. "Miniature curved artificial compound eyes". In: Proceedings of the National Academy of Sciences 110.23 (2013), pp. 9267-9272.
[7] Bruno Herissé et al. "Landing a VTOL unmanned aerial vehicle on a moving platform using optical flow". In: IEEE Transactions on robotics 28.1 (2011), pp. 77-89.

[8] Hann Woei Ho, Guido CHE de Croon, and Qiping Chu. "Distance and velocity estimation using optical flow from a monocular camera". In: International Journal of Micro Air Vehicles 9.3 (2017), pp. 198-208.

[9] Fumiya Iida. "Biologically inspired visual odometer for navigation of a flying robot". In: Robotics and autonomous systems 44.3-4 (2003), pp. 201-208.

[10] Farid Kendoul, Isabelle Fantoni, and Kenzo Nonami. "Optic flow-based vision system for autonomous 3D localization and control of small aerial vehicles". In: Robotics and autonomous systems 57.6-7 (2009), pp. 591-602.

[11] WH Kirchner and MV Srinivasan. "Freely flying honeybees use image motion to estimate object distance". In: Naturwissenschaften 76.6 (1989), pp. 281-282.

[12] Karl Kral. "Side-to-side head movements to obtain motion depth cues:: A short review of research on the praying mantis". In: Behavioural Processes 43.1 (1998), pp. 71-77.

[13] S Mafrica, A Servel, and F Ruffier. "Minimalistic optic flow sensors applied to indoor and outdoor visual guidance and odometry on a car-like robot." In: Bioinspiration \& biomimetics 11.6 (2016), p. 066007.

[14] Richard JD Moore et al. "A stereo vision system for uav guidance". In: 2009 IEEE/RSJ International Conference on Intelligent Robots and Systems. IEEE. 2009, pp. 3386-3391.

[15] Sergiu Nedevschi et al. "High accuracy stereo vision system for far distance obstacle detection". In: IEEE Intelligent Vehicles Symposium, 2004. IEEE. 2004, pp. 292-297.

[16] Geoffrey Portelli et al. "Honeybees' speed depends on dorsal as well as lateral, ventral and frontal optic flows". In: PloS one 6.5 (2011), e19486.

[17] Frédéric L Roubieu et al. "Two-Directional 1-g Visual Motion Sensor Inspired by the Fly' s Eye". In: IEEE Sensors 13.3 (2013), pp. 1025-1035.

[18] Franck Ruffier and Nicolas Franceschini. "Optic flow regulation: the key to aircraft automatic guidance". In: Robotics and Autonomous Systems 50.4 (2005), pp. 177-194.

[19] Guillaume Sabiron et al. "Low-speed optic-flow sensor onboard an unmanned helicopter flying outside over fields". In: Proceedings - IEEE International Conference on Robotics and Automation (2013), pp. 1742-1749. ISSN: 10504729. DOI: $10.1109 /$ ICRA.2013.6630806.

[20] Ashutosh Saxena, Jamie Schulte, Andrew Y Ng, et al. "Depth Estimation Using Monocular and Stereo Cues." In: IJCAI. Vol. 7. 2007, pp. 2197-2203.

[21] Julien R Serres and Franck Ruffier. "Optic flow-based collision-free strategies: From insects to robots". In: Arthropod structure \& development 46.5 (2017), pp. 703-717.

[22] Floris Van Breugel, Kristi Morgansen, and Michael H Dickinson. "Monocular distance estimation from optic flow during active landing maneuvers". In: Bioinspiration \& biomimetics 9.2 (2014), p. 025002.

[23] Erik Vanhoutte et al. "Time-of-travel methods for measuring optical flow on board a micro flying robot". In: Sensors 17.3 (2017), p. 571. 


\section{A. Appendix: The Extended Kalman Filter}

The discretized model of the test bench can be expressed as

$$
\left\{\begin{array}{c}
X[k+1]=\Phi \cdot X[k]+\Gamma \cdot u[k] \\
y[k]=C_{k} \cdot X[k]+D_{k} \cdot u[k]
\end{array}\right.
$$

with

$$
\begin{gathered}
\Phi=e^{A \cdot d t} \\
\Gamma=\left(\int_{0}^{d t} e^{A \cdot \tau} d \tau\right) \cdot B=\left(A^{T} \cdot e^{A \cdot d t}-A^{T}\right) \cdot B \\
C_{k}=h\left(x_{k}\right)=\left[\frac{x_{2}[k]}{x_{1}[k]}\right] \\
D_{k}=0
\end{gathered}
$$

where $d t$ is the discretization time.

To estimate the distance $h$ from the panorama, the EKF took the following iterative steps for each $k^{\text {th }}$ time

\section{Prediction step}

(a) One-step ahead prediction

$$
X_{k / k-1}=\Phi \cdot X_{k-1 / k-1}+\Gamma \cdot u_{k-1 / k-1}
$$

(b) Covariance matrix of the state prediction error vector

$$
P_{k / k-1}=\Phi \cdot P_{k-1 / k-1} \cdot \Phi^{T}+Q
$$

\section{Correction step}

(c) Measurement update

$$
X_{k / k}=X_{k / k-1}+K_{k} \cdot\left(y_{k}-H_{k} \cdot X_{k / k-1}\right)
$$

with $K_{k}$ Kalman gain defined as

$$
K_{k}=P_{k / k-1} \cdot H_{k}^{T} \cdot\left[H_{k} \cdot P_{k / k-1} \cdot H_{k}^{T}+R_{k}\right]^{-1}
$$

and $H_{k}$ Jacobian matrix for the non linear function defined as follows

$$
H_{k}=\left.\frac{\partial h}{\partial X}\right|_{X=X_{k / k-1}}=\left[\begin{array}{ll}
-\frac{\dot{x}}{x^{2}} & \frac{1}{x}
\end{array}\right]
$$

(d) Covariance matrix of state estimation error vector

$$
P_{k / k}=P_{k / k-1}+K_{k} \cdot\left[H_{k} \cdot P_{k / k-1} \cdot H_{k}^{T}+R_{k}\right] \cdot K_{k}^{T}
$$

(e) Innovation

$$
\tilde{y}_{k}=y_{k}-H_{k} \cdot x_{k / k}
$$

